\title{
Autoimmune disease, familial clustering and thyroid carcinoma coexistent with autoimmune thyroiditis in children and adolescence: A cross-sectional study from the Czech Republic
}

\author{
Renata Pomahacova, Jana Zamboryova, Petra Paterova, Karel Fiklik, Zdenka Cerna, Vaclav Lad, Eva Skalicka, \\ Michal Huml, Josef Sykora
}

Background. The prevalence of autoimmune thyroiditis (AIT), as the most common autoimmune disease (AD) and papillary thyroid cancer (PTC) is steadily rising in children. The aim of this study was to determine the coexistence of other AD and thyroid carcinoma (TC) in AIT.

Methods. The cross-sectional study conducted at a tertiary center comprised AIT children ( $<19$ years). Data on age/ sex, thyroid function and ultrasound, autoantibodies, associated AD, familial occurence of $A D$ and the occurence of TC for each child were collected.

Results. In total, 231 eligible patients (77\% females) were included. The most common onset (66\%) was during adolescence. At onset, hypothyroidism was detected in $59.3 \%$; hashitoxicosis in $1.3 \%$. The positivity of both autoantibodies was present in $60.6 \%$, the negativity was in 3,5\%. We confirmed a high frequency (44.6\%) of AD with AIT predominance in parents and/or grandparents of patients and in siblings (7.4\%). 15.2\% had at least 1 comorbid AD, of which type 1 diabetes mellitus was the most common (8.5\%). Over a period of 7 years TC was diagnosed in 16 patients (mean age 13.5 years) with predominance of PTC in 15 (94\%) patients. AIT had concurrently $69 \%$ patients. $56 \%$ of patients had metastases (89\% in AIT subjects). An invasive PTC was present in $44 \%$ ( $86 \%$ in AIT subjects).

Conclusions. The prevalence rate of AD in AIT and first-degree relatives is high, and several new associations have been reported. Providers should be aware of comorbidities and TC in AIT as this would help in early diagnoses and timely interventions.

Key words: autoimmune thyroiditis, associated autoimmune diseases, familial occurence of autoimmune diseases, prevalence, papillary thyroid carcinoma

Received: December 21, 2017; Accepted with revision: May 23, 2018; Available online: June 7, 2018 https://doi.org/10.5507/bp.2018.030

Department of Paediatrics, Charles University in Prague, Faculty of Medicine in Pilsen, Czech Republic Corresponding author: Renata Pomahacova, e-mail:pomahacova@fnplzen.cz

\section{INTRODUCTION}

Autoimmune thyroiditis (AIT), or chronic lymphocytic thyroiditis (CLT), is the most common thyroid disorder and autoimmune disease in the pediatric age range, affecting preferentially females, at any age of life. Graves disease (GD) is less frequent. The most common age at presentation of AIT is puberty and adolescence.Both a goitrous (Hashimoto's thyroiditis) and a nongoitrous (atrophic thyroiditis) variant of AIT have been identified ${ }^{1}$.

Approximately $70 \%$ of disease risk has been attributed to genetic background with environmental factors being important in triggering the disease in susceptible individuals. Probably polymorphisms in multiple genes play a role ${ }^{1}$.

Thyroid peroxidase (TPO) antibodies (Abs) and thyroglobulin $(\mathrm{Tg})$ are valuable markers of underlying autoimmune thyroid destruction. Thyroid-stimulating hormone (TSH) receptor blocking Abs are found in 18\% of children and adolescents with severe hypothyroidism and, when persistent, may identify adolescent females at risk of having babies with transient congenital hypothyroidism². Altered thyroid echostructure with hypoechogenicity on thyroid ultrasonography is typical of AIT. There are scarce data on the coexistence of papillary thyroid carcinoma (PTC) in AIT in pediatric patients ${ }^{3-5}$.

Thyroid function tests show variations at the time of diagnosis: mostly euthyroid or hypothyroid and rarely hyperthyroid. Severe hypothyroidism may lead to retardation in growth and development ${ }^{6,7}$.

Patients with AIT are widely held to often have other $\mathrm{AD}$, organ-specific and non-organ-specific e.g. rheumatoid arthritis or systemic lupus erythematosus (SLE). Organspecific $\mathrm{AD}$, such as AIT, may also be part of the autoimmune polyendocrine syndrome (APS) $\left(\right.$ ref. $\left.^{8-11}\right)$. Little is known, however, of the familial occurence and clustering of autoimmune diseases (AD) and coexistence of autoimmunity in AIT subjects ${ }^{9,10,12,13}$.

In addresing the gap in knowledge, the aim of the study was (1) to comprehensively analyse the clinical profile and the autoimmune comorbidities in pediatric subjects with AIT, (2) to recognize the occurrence of 
thyroid carcinoma (TC) regarding AIT, from the same geographical area.

\section{PATIENTS AND METHODS}

\section{Description of subjects}

We retrospectively analyzed data from a contemporary group of patients aged between 3 and 18 years, receiving full care in the Outpatient Department of Endocrinology at the Department of Pediatrics of the Faculty Hospital in Pilsen. There were autoimmune thyreopathies in 246 children, GD in 15 children and AIT in 231 children.

\section{Methods}

This was a cross-sectional, single-institution study. Information collected from chart review included demographic data on thyroid function, positivity of thyroidspecific antibodies at the time of diagnosis, thyroid ultrasound scan, occurence of associated $\mathrm{AD}, \mathrm{AD}$ in first degree relatives of patients, and the occurence of TC. Children were included if they had the diagnosis of AIT by the positivity of specific TPO Abs and/or Tg Abs, altered thyroid echostructure with hypoechogenicity and irregular texture on thyroid ultrasonography. The thyroid function was determined by TSH and fT4 (free thyroxin) analyses. The diagnosis of TC was based on FNAB (fine needle aspiration biopsy) and on histological examination of thyroid nodules. Disorders were considered to be of autoimmune origin if they were included in the list of AD reported by Hyter et al. ${ }^{14}$.

\section{Ethics}

As the study sample simply analysed data that was routinely collected as part of the clinical care of the patients with AIT, the study did not require informed consent and was approved as an evaluation of health care delivery. All procedures of this study were in compliance with the Declaration of Helsinky.

\section{Statistical analysis}

We used standard descriptive statistics to summarize the characteristics of the entire study population. Unless stated otherwise, data are presented as means \pm standard deviations (SDs) for continuous variables, and percentage for categorical data (\%). Analyses for comparison between different groups were performed by the Wilcoxon rank sum test and the Chi-square test as appropriate. Statistical analyses were done using SPSS version 14.0 for Windows (SPSS Inc., Chicago, IL, USA). The accepted level of significance for all analyses was $P<0.05$.

\section{RESULTS}

\section{Demographic data of analysed AIT subjects at the time of diagnosis}

The baseline characteristics are shown in Table 1 . The subjects were predominantly (98\%) of Czech ethnic background in our study. A total of 231 eligible individuals
Table 1. Demographic characteristics of patients with AIT at the time of diagnosis.

\begin{tabular}{lc}
\hline Demographics & overall $(\mathrm{n}=231)$ \\
\hline Mean age, y (range) & $13(3-18)$ \\
Gender & \\
Female & $177(77 \%)$ \\
$\quad$ Male & $54(23 \%)$ \\
Race, ethnicity & \\
White & $226(98 \%)$ \\
Other & $5(2 \%)$ \\
Unknown & 0 \\
\hline
\end{tabular}

with AIT including 4 patients with Turner syndrome and their parents participated in the study. AIT was found more frequently in girls (77\%) than in boys (the ratio of male cases to female cases was 1: 3.5). As a group, age distribution of the AIT group at onset: 79 children (34\%) were under 10 years of age, of whom $14(6 \%)$ children were under 6 years of age; 152 children (66\%) were aged 11-18 years (puberty and adolescence).

\section{Comparison age, age of onset, and duration of AIT between AIT cases with and without coexisting AD}

Statistical analysis revealed no significant differences between those with and without a history of at least one comorbid AD in onset of AIT, current age, and duration.

\section{Thyroid function in AIT patients at the time of diagnosis}

Regarding the thyroid function at the time of AIT diagnosis, 137 children presented with hypothyroidism (59.3\%), 91 (39.4\%) patients had normal thyroid function and $3(1.3 \%)$ subjects had thyreotoxicosis and following transition to hypothyroidism (hashitoxicosis). 72 patients $(52.6 \%)$ presented subclinical hypothyroidism with TSH 4.2-10 mIU/L (normal range 0.3-4) and 65 children $(47.4 \%)$ had clinical signs of hypothyroidism. Among the children with clinically manifested hypothyroidism, there were 30 patients (46\%) with TSH > $50 \mathrm{mIU} / \mathrm{L}$, myxoedema, and eight children also had growth disorder with growth deceleration. Five children with severe hypothyroidism had atrophic thyroiditis with negative TSH receptor blocking Abs.

\section{Thyroid antibodies in AIT patients at the time of diagnosis}

There was a positivity of both TPO and Tg Abs in 140 patients $(60.6 \%)$. In 47 children $(20.3 \%)$ only $\mathrm{Tg}$ Abs were positive, and 36 children (15.6\%) had just TPO Abs. Thyroid autoantibodies were negative in 8 patients with AIT (3.5\%).

\section{Coexistence of $\mathrm{AD}$ in AIT patients and firts-degree relatives}

56 children $(24.2 \%)$ in the group of 231 AIT children had positive allergic anamneses, most of them had polyvalent allergy (95\%). AD in parents, grandparents or siblings in the group of 231 AIT children was detected in 103 children (44.6\%). Only in three cases $(1.3 \%)$ was there $\mathrm{AD}$ in a male parent or grandparent. In the 
patients ' relatives there was AIT in 74 cases $(71.8 \%)$, GD in 16 cases $(15.5 \%)$ and other $\mathrm{AD}$ in 13 cases $(12.7 \%)$ (SLE, rheumatoid arthritis, coeliac disease - CD, type 1 diabetes mellitus - T1DM, vitiligo). In the 231 AIT children there were 17 patients (7.4\%) who had siblings with AD. The most common AD in siblings was AIT (Table 2). In all, $35(15.2 \%)$ subjects (68\% female) in a contemporary group of children affected with AIT had a history of 1 or more associated AD (Table 3). T1DM was the most common comorbid disease, seen in 19 (8.5\%) patients with AIT. One patient with T1DM was diagnosed with APS type 2. Of note, in 5.5\% of the cases, another AD of non-endocrine organs was first diagnosed, and then, after several years, the diagnosis of AIT with hypofunction of the thyroid gland was established.

\section{Coexistence of $\mathrm{AD}$ in $\mathrm{AIT}$ patients by gender}

The occurence of AD was not statistically significant for gender. Of 177 in toto female patients with AIT, 24 (13.6\%) had comorbid AD compared with 11 (20.3\%) of 54 in toto male patients with AIT with comorbid AD $(P=0.31)$. Of 177 total female subjects with AIT, $12(6.7 \%)$ had T1DM compared with 7 (12.9\%) of 54 total male patients with AIT with T1DM $(P=0.29)$. There were no significant differences in prevalence of any other AD between genders.

\section{Coexistence of AIT and thyroid carcinoma in analysed AIT group}

TC was diagnosed over the 7-year study period in 16 patients including PTC in 15 patients (94\%) and follicular carcinoma in 1 patient $(6 \%)$. There were 13 patients of female sex $(81 \%)$ and 3 patients of male sex (19\%). The mean age was 13.5 years ranging from 10 to 18 years. 11 PTC patients (69\%) concurrently had AIT and 5 patients (31\%) had no signs of AIT. The diagnosis of PTC in 6 AIT patients was based on periodic ultrasonography follow-up (papillary microcarcinoma was present in 2 patients) and in 5 patients the diagnosis of PTC was confirmed at the same time as the diagnosis of AIT. 9 patients (56\%) had metastases (lymf node in the cervical area, mediastinum in 2 patients, second lobe of the thyroid gland in two patients, lungs in 1 patient); AIT was present in 8 out of these nine patients (89\%). Extracapsular invasion of PTC was present in seven out of 16 patients (44\%); AIT was present in six out of these 7 patients $(86 \%) .4$ patients had papillary microcarcinoma.

\section{DISCUSSION}

This is one of the most comprehensive studies to date to identify the clinical profile, comorbid AD and TC in cases with well-characterized paediatric AIT. To the best of our knowledge, this is the first Czech paediatric study covering comorbidities and TC in AIT. The major findings are that in AIT patients: (a) the comorbidity rate of AD in AIT patients is high with a high prevalence of AD in first-degree relatives with a predominance of AIT as the most frequent $\mathrm{AD},(\mathrm{b})$ we highlighted the importance of screening for AIT in subjects from genetic predisposed families to AD and screening for other AD in AIT, (c) the most common onset of AIT was in puberty and adolescence with the majority of female sex, and (d) AIT may be a risk factor for development of PTC, predominantly invasive form of PTC.

Our observations and available pediatric data suggest that AIT occurs more frequently in females ${ }^{1}$. Furthermore, this fact was also confirmed by the family history analysis in the AIT group, where only $1.3 \%$ of parents/grandparents of male sex suffered from AD. This study found that the most common age at presentation was puberty. On the other hand, the disease may occur rarely in very young pre-school children. Thus, we pointed out that this fact should not be underestimated ${ }^{1}$.

The findings of this study further illustrate, that in children with AIT, TPO and Tg Abs are present in the majority of patients. TSH receptor blocking Abs are found in $18 \%$ of children and adolescents with severe hypothyroidism, which may lead to transient congenital hypothyroidism $^{2}$. The positivity of both specific autoantibodies (TPO and $\mathrm{Tg}$ Abs) was present in $60.6 \%$ of patients at the time of AIT diagnosis, the negativity of both autoantibodies was present in $3.5 \%$ of patients with AIT, which does not exclude this diagnosis. We emphasize the importance of the investigation of TSH receptor blocking Abs especially in female sex patients with the atrophic form of AIT in pregnancy. The positivity of these antibodies predict the possibility of blocking the thyroid gland in foetus resulting in development of transient congenital hypothyroidism ${ }^{15}$. To add further to the complexity, we suggest that the comorbidity rate of alergic reactions in AIT was high. We found $24 \%$ of AIT patiens with a positive allergic history confirming genetic predisposition to immunopathological reactions in these individuals. For that reason, screening of AIT in children with allergy could be benefical.

The familial occurence of AD is available from only a few studies with conflicting results9,10,12,13. A notable percentage of parents and/or grandparents of our patients with AIT had AD (44.6\%) suggesting genetic predisposition to autoimmune immunopathological reaction in such affected families probably depending on the population studied. The most commonly associated conditions were $\mathrm{AD}$ of the thyroid gland.-This is similar to the results by Fallahi et al. ${ }^{10}$. The prevalence of AIT of our patients ' relatives was $72 \%$, and the prevalence of GD was $15.5 \%$. The prevalence of AD in siblings of our AIT patients was $7,4 \%$, with predominance of AIT too (Table 2). Compared with other studies, Segni et al. observed the

Table 2. Frequencies of autoimmune diseases in AIT patient's siblings.

\begin{tabular}{lc}
\hline $\begin{array}{l}\text { Autoimmune diseases in AIT } \\
\text { patients' siblings (7.4\%) }\end{array}$ & $\begin{array}{c}\text { Number } \\
\text { of siblings (\%) }\end{array}$ \\
\hline Chronic lymphocytic thyroiditis & $12(5.3)$ \\
Type 1 diabetes & $2(0.9)$ \\
Graves disease & $1(0.4)$ \\
Idiopathic thrombocytopenic purpura & $1(0.4)$ \\
Sclerodermia circumscripta & $1(0.4)$ \\
\hline
\end{tabular}


prevalence of AIT in siblings of affected children with autoimmune thyroid diseases in $27 \%$ (ref. ${ }^{13}$ ). Based on our findings, screening for these conditions, especially AIT, should be considered in first - degree relatives.

At the time of AIT diagnosis, $59 \%$ of our children had already hypofunction of the thyroid gland, $46 \%$ of whom had severe hypothyroidism. In some cases, the affected children were from families with genetic disposition to AD. This fact leads to the issue of the necessity of periodic thyroid function follow-up of individuals in such affected families due to possible development of AD. Severe primary hypothyroidism can have permanent aftereffects on the childs' growth and development ${ }^{6,7}$.

An important question that has not been fully addressed so far is the prevalence of AD in children with AIT (ref. ${ }^{9-11}$ ), but a study conducted in the Czech Republic is lacking. In the current study, we clearly illustrate associations between AIT and other AD. Additionally, our study provides evidence for several new $\mathrm{AD}$ as provided in Table 3. However, it is important to realise that although case reports of association of these disorders are in existence, these conditions have rarely or never been reported in a large cohort of pediatric patients suffering from AIT. In our trial, we found that AIT may occur alone or may coexist with other comorbid AD (15.2\% patients), particularly T1DM. The complex pathophysiology behind the development of AD including T1DM seen in AIT still remains a challenge. In this context, however, as clinical effects of $\mathrm{AD}$ are heterogenous and are probably influenced by the various underlying cause, a reliable prediction how many persons develop other $\mathrm{AD}$ is not possible. It is of particular note that in nearly $6 \%$ of the cases, another AD of non-endocrine organs was first diagnosed, and after several years, they developed AIT with hypofunction of the thyroid gland. Thus, based on the literature and our findings, we recommend searching for AIT in patients with other endocrine AD and non-endocrine AD.

There is a lot of controversy related to the role of AIT in pediatric TC. However, there is still much to be learned about the coexistence of AIT and PTC. It is believed that AIT and PTC share some common epidemiological features, such as the relationship to ionizing radiation exposure and dietary iodine, as well as some molecular features. The oncogenic RET/PTC rearrangements, a specific genetic alteration in PTC patients, can be also found in AIT. Whether the prevalence of PTC is higher in children with AIT has not been fully addressed ${ }^{9,16-19}$. Iliadon et al. reports the prevalence of invasive form of differentiated thyroid carcinoma in children and adolescents with AIT ( ref. $^{19}$ ). We diagnosed TC in 16 patients in the evaluation period of 7 years with predominance of PTC in 15 patients. According to published data, we also confirmed the majority of female sex $(81 \%)$. The mean age was 13.5 years, and the youngest child was 10 years old. One of our main study finding was that the majority of patients concurrently had AIT. The diagnosis of PTC in six AIT patients was based on periodic ultrasonography follow-up and papillary microcarcinoma was present in two of these patients. In five patients the diagnosis of PTC was confirmed at the same time as the diagnosis of AIT.
Table 3. Frequencies of autoimmune diseases in AIT cohort observed.

\begin{tabular}{lc}
\hline $\begin{array}{l}\text { Associated autoimmune diseases } \\
(15.2 \%)\end{array}$ & $\begin{array}{c}\text { Number } \\
\text { of AIT patients (\%) }\end{array}$ \\
\hline Type 1 diabetes & $19(8.5)$ \\
(APS 2 - 1 patient) & $3(1.3)$ \\
Coeliac disease & $3(1.3)$ \\
Crohn's disease & $1(0.4)$ \\
Ulcerative colitis & $1(0.4)$ \\
Systemic lupus erythematosus & $1(0.4)$ \\
Sjögren's syndrome & $1(0.4)$ \\
Juvenile idiopathic arthritis & $1(0.4)$ \\
Vitiligo & $1(0.4)$ \\
Sclerodermia circumscripta & $2(0.9)$ \\
Alopecia & $1(0.4)$ \\
Idiopathic thrombocytopenic purpura & $1(0.4)$ \\
Autoimmune hemolytic anemia & \\
\hline
\end{tabular}

APS 2, autoimmune polyglandular syndrome type 2

$56 \%$ of patients out of our analysed group had metastases and AIT was present in the majority (89\%) of these patients. An invasive form of PTC was present in $44 \%$ of patients, predominantly in patients with AIT (86\%) too. Patients with papillary microcarcinoma underwent total thyreoidectomy without radioactive iodine treatment. Thus, our findings have important implications for clinical practice. We felt it legitimate to assume that AIT may be a risk factor for development of PTC in pediatric population. In agreement with published data, we suggest that AIT patients should be regularly followed up for the appearance of nodules by regular neck ultrasonography assessement ${ }^{9}$.

\section{CONCLUSION}

We observed that AD tend to coexist in the same individuals and to cluster in families with AIT. A routine screening strategy should be implemented with the determination AIT as the most common AD in genetically predisposed families to $\mathrm{AD}$, in patients with other $\mathrm{AD}$ and the follow-up of the development of organ specific autoantibodies in patients with diagnosis of AD in order to make an early diagnosis of pre-clinical period of serious endocrinopathies. Our data suggest that AIT may be a risk factor of PTC, and patients should be regularly, once a year, monitored for the appearance of thyroid nodules by neck ultrasonography. Further studies may help uncover the sequence of development of $\mathrm{AD}$ and TC in AIT.

\section{ABBREVIATION}

AIT, Autoimmune thyroiditis; CLT, Chronic lymphocytic thyroiditis; AD, Autoimmune disease (AD); PTC, Papillary thyroid cancer; TC, Thyroid carcinoma; GD, Graves disease; T1DM, Diabetes mellitus type 1; SLE, Systemic lupus erythematosus; APS, Autoimmune poly- 
endocrine syndrome; CD, Coeliac disease; TPO, Thyroid peroxidase; Tg, thyroglobulin; Abs, Antibodies; fT4, Free thyroxin; TSH, Thyroid-stimulating hormone; FNAB, Fine needle aspiration biopsy.

Acknowledgement: This work was supported by a Project of the Charles University Research Fund (Progres Q-39). Author contributions: RP, JZ: contributed equally to this work; RP, JZ, PP, KF, ZC, VL, MH, JS: designed the research and concept, recruited subjects and performed acqusition of data; RP, JZ, VL, ES, MH, JS: analyzed and interpreted the data and drafted the manuscript; RP, JS: wrote the manuscript; RP, JZ, KF, ZC, VL, ES, MH, JS: drafted the manuscript, critically revised the manuscript for important intelectuall content; All authors approved the final version of the manuscript.

Conflict of interest statement: The authors state that there are no conflicts of interest regarding the publication of this article.

\section{REFERENCES}

1. Brown RS. Autoimmune thyroiditis in childhood. J Clin Res Pediatr Endocrinol 2013;5(S1):5-9. doi:10.4274/Jcrpe.855

2. Feingold SB, Smith J, Houtz J, Popovsky E, Brown RS. Prevalence and functional significance of thyrotropin receptor blocking antibodies in children and adolescents with chronic lymphocytic thyroiditis. J Clin Endocrinol Metab 2009;94:4742-8.

3. Skarpa V, Kousta E, Tertipi A, Anyfandakis K, Vakaki M, Dolianiti M, Fotinou A, Papathanasiou A. Epidemiological characteristics of children with autoimmune thyroid disease. Hormones (Athens) 2011;10(3):207-14.

4. Feldt-Rasmussen U, Rasmussen AK. Autoimmunity in differentiated thyroid cancer: significance and related clinical problems. Hormones (Athens) 2010;9(2):109-17.

5. Mazokopakis EE, Tzortzinis AA, Dalieraki-Ott El, Tsartsalis AN, Syros PK, Karefilakis CM, Papadomanolaki MG, Starakis IK. Coexistence of Hashimoto's thyroiditis with papillary thyroid carcinoma. A retrospective study. Hormones (Athens) 2010;9(4):312-7.

6. Özen S, Berk Ö, Şimşek DG, Darcan S. Clinical course of Hashimoto's thyroiditis and effects of levothyroxine therapy on the clinical course of the disease in children and adolescents. J Clin Res Pediatr Endocrinol 2011;3(4):192-7.
7. Radetti G, Gottardi E, Bona G, Corrias A, Salardi S, Loche S; Study Group for Thyroid Diseases of the Italian Society for Pediatric Endocrinology and Diabetes (SIEDP/ISPED) The natural history of euthyroid Hashimoto's thyroiditis in children. J Pediatr 2006;149(6):82732.

8. Kakleas K, Soldatou A, Karachaliou F, Karavanaki K. Associated autoimmune diseases in children and adolescents with type 1 diabetes mellitus (T1DM). Autoimmun Rev 2015;14(9):781-97.

9. Antonelli A, Ferrari SM, Corrado A, Di Domenicantonio A, Fallahi P. Autoimmune thyroid disorders. Autoimmun Rev 2015;14(2):174-80.

10. Fallahi P, Ferrari SM, Ruffilli I, Elia G, Biricotti M, Vita R, Benvenga S, Antonelli $A$. The association of other autoimmune diseases in patients with autoimmune thyroiditis: Review of the literature and report of a large series of patients. Autoimmun Rev 2016;15(12):1125-8.

11. Głowińska-Olszewska B, Michalak J, Łuczyński W, Del Pilar Larosa M, Chen S, Furmaniak J, Smith BR, Bossowski A. Organ-specific autoimmunity in relation to clinical characteristics in children with long-lasting type 1 diabetes. J Pediatr Endocrinol Metab 2016;29(6):647-56.

12. Dittmar M, Libich C, Brenzel T, Kahaly GJ. Increased familial clustering of autoimmune thyroid diseases. Horm Metab Res 2011;43(3):200-4

13. Segni M, Wood J, Pucarelli I, Toscano V, Toscano R, Pasquino AM. Clustering of autoimmune thyroid diseases in children and adolescents: a study of 66 families. J Pediatr Endocrinol Metab 2001;14(5):1271-5.

14. Hayter SM, Cook MC. Updated assessment of the prevalence, spectrum and case definition of autoimmune disease. Autoimmun Rev 2012;11:754-65.

15. Brown RS, Alter CA, Sadeghi-Nejad A. Severe unsuspected maternal hypothyroidism discovered after the diagnosis of thyrotropin receptor-blocking antibody-induced congenital hypothyroidism in the neonate: failure to recognize and implications to the fetus. Horm Res Paediatr 2015;83(2):132-5.

16. Lee JH, Kim Y, Choi JW, Kim YS. The association between papillary thyroid carcinoma and histologically proven Hashimoto's thyroiditis: a meta-analysis. Eur J Endocrinol 2013;168(3):343-9.

17. Liu X, Zhu L, Cui D, Wang Z, Chen H, Duan Y, Shen MP, Zhang ZH, Wang XD, Chen JW, Alexander EK, Yang T. Coexistence of Histologically Confirmed Hashimoto's Thyroiditis with Different Stages of Papillary Thyroid Carcinoma in a Consecutive Chinese Cohort. Int J Endocrinol 2014:2014:769294. doi:10.1155/2014/769294

18. Konturek A, Barczyński M, Wierzchowski W, Stopa M, Nowak W. Coexistence of papillary thyroid cancer with Hashimoto thyroiditis. Langenbecks Arch Surg 2013;398(3):389-94.

19. Iliadou PK, Effraimidis G, Konstantinos M, Grigorios $P$, Mitsakis $P$ Patakiouta F, Pazaitou-Panayiotou K. Chronic lymphocytic thyroiditis is associated with invasive characteristics of differentiated thyroid carcinoma in children and adolescents. Eur J Endocrinol 2015;173(6):827-33. 